\title{
Propuesta para el desarrollo del pensamiento sistémico en los programas de formación de posgrado del convenio USTA-ICONTEC
}

Proposal for the development of systemic thinking in the postgraduate training programs of the USTA-ICONTEC Convention

Proposta para o desenvolvimento do pensamento sistêmico nos programas de formação de pós-grraduação do convênio USTA-ICONTEC

Liliana Muños Pineda**

Consultora independiente 


\section{RESUMEN}

Esta investigación busca establecer la relación entre el pensamiento sistémico y la gestión integral para su posible incorporación en los programas de posgrado del convenio USTA-ICONTEC. Se partió de una revisión sistemática exploratoria de artículos publicados en las bases de datos Science Direct, Ebscohost, Jstor y SciVerse Scopus, entre 2005 y 2015, usando términos MeSH pensamiento sistémico y gestión. Seguido a ello, con el objetivo de conocer las concepciones de las partes interesadas relevantes, es decir, las que directamente están inmersas en el proceso de formación del convenio, se realizaron entrevistas con los docentes, los egresados y los estudiantes de los programas de posgrados, posteriormente, se realizó un análisis de contenido de los aspectos curriculares. Finalmente, se efectuó una revisión de las tres fuentes enunciadas y a partir de la triangulación metodológica de estos hallazgos, se definió la estrategia para el desarrollo del pensamiento sistémico en la formación del estudiante de posgrado de sistemas de gestión, que se propone sea incorporada en la actualización curricular de los programas.

Palabras clave: pensamiento sistémico, gestión integral, gestión integrada, organizaciones.

\section{ABSTRACT}

This research seeks to establish the relationship between systemic thinking and integral management for its possible inclusion in the postgraduate programs of the USTA-ICONTEC agreement. It was based on an exploratory systematic review of articles published in the databases Science Direct, Ebscohost, Jstor and SciVerse Scopus, between 2005 and 2015, using $\mathrm{MeSH}$ systemic thinking and management terms. Following this, interviews with teachers, graduates and students of the postgraduate programs were carried out, in order to know the conceptions of the relevant stakeholders. Later, a content analysis of the curricular aspects was carried out. Finally, a review of the three sources and from the methodological triangulation of these findings, defined the strategy for the development of systemic thinking in the postgraduate student training management systems, which is proposed to be incorporated in the update curricular of the programs.

Keywords: systems thinking, integral management, integrated management, organizations.

\section{RESUMO}

Esta pesquisa visa estabelecer a relação entre 0 pensamento sistêmico e a gestão integrada para sua possível inclusão nos programas de pós-graduação do convênio USTA-ICONTEC. Partiu-se de uma revisão sistemática e exploratória dos artigos publicados nas bases de dados Science Direct, Ebscohost, Jstor e SciVerse Scopus, entre 2005 e 2015, utilizando termos MeSH pensamento sistêmico e gestão. Seguido a isto, com o objetivo de conhecer as concepções das partes interessadas relevantes, ou seja, as que estão diretamente envolvidas no processo de formação do Convênio, foram realizadas entrevistas com professores, graduados e estudantes dos programas de pós-graduação, posteriormente, uma análise do conteúdo dos aspectos curriculares. Finalmente, efetuou-se uma revisão das três fontes listadas e a partir da triangulação metodológica destes achados, se definiu a estratégia para o desenvolvimento do pensamento sistêmico na formação do estudante de pós-graduação de sistemas de gestão, que se propõe ser incorporado na atualização curricular dos programas.

Palavras-chave: pensamento sistêmico, gestão integral, gestão integrada, organizações. 


\section{INTRODUCCIÓN}

Un número importante de organizaciones han sido certificadas con las normas técnicas ISO. En primer lugar se encuentra la ISO 9001, desde 1988 y hasta 2015 más de un millón de organizaciones han recibido este reconocimiento; seguida de la ISO 14001, con más de 300 mil certificaciones a nivel mundial. La gran mayoría de las organizaciones buscan cumplir unos estándares mínimos y satisfacer las necesidades de las partes interesadas, no obstante, en muchas de ellas se mantienen las estructuras verticales lo cual impide que sean concebidas integralmente, es decir, como sistema. En este sentido, se requiere la formación de profesionales, especialistas y magísteres con un pensamiento crítico y sistémico que le ayuden a las organizaciones a cambiar esta realidad.

En los antecedentes del contexto mundial se encuentra escasa información que relacione el pensamiento sistémico y la gestión integral en las organizaciones, entendida esta gestión integral como la armonización de la organización con los ejes del desarrollo sostenible: la creación de riqueza, la protección del medio ambiente y la equidad social. No obstante, en correspondencia con el pensamiento sistémico, se puede referenciar la investigación de Gallegos (2016), quien hace una reseña de la obra de Morin y analiza 413 artículos que, basados en el pensamiento complejo, abordan temáticas como educación, sociología, epistemología, cultura, salud, ética y ecología, entre otras; concluye que el área donde hay mayor concentración de estudios relacionados con complejidad es la educación, donde se encuentran trabajos vinculados con sistemas curriculares, didáctica en la enseñanza, formación de profesores, pedagogía empleada en clases y educación en valores.

El autor indica que la complejidad proporciona elementos teóricos y metodológicos para avanzar en la mejora de la educación, además con mayor frecuencia en dichos documentos son citados los principios de ho- logramática, recursividad y dialógica. Agrega que aún no se ha desarrollado una pedagogía de la complejidad y que a pesar de que este importante número de estudios proponen modelos teóricos no se ve que haya avances importantes en implementación.

Se observa que no existen documentos conocidos que estudien la relación existente entre el pensamiento sistémico y la gestión integral, no obstante, dada la generalidad del pensamiento sistémico, aplica para todas las áreas del conocimiento así como para la implementación de sistemas de gestión normalizados 0 integrales al interior de las organizaciones.

Al respecto, Ackoff (2007) menciona que un sistema es un todo y que este no puede ser dividido en partes independientes, dado que perdería sus propiedades internas, lo que lleva a comprender el rol de la gerencia como la administración de las interacciones entre las partes del sistema y no solo como las acciones. Los sistemas son de tres tipos: 1) sistema mecánico, ni él ni sus partes tienen un propósito propio, pueden servir a una entidad ajena, el automóvil es un ejemplo; 2) sistema orgánico, tiene como mínimo un propósito, aun cuando sus partes tienen funciones que sirven al propósito del todo, pueden ser esenciales como los pulmones o no esenciales como las uñas y, 3) sistema social, es un sistema abierto que tiene propósitos propios, forma parte de otro sistema mayor, un ejemplo son las organizaciones; precisando que un sistema que involucre personas en todo caso debe ser considerado como sistema social.

En nuestra cultura los directivos de las organizaciones son ilustrados para asumir que el desempeño de las organizaciones (sistemas sociales) puede ser mejorado optimizando el desempeño de cada una de las partes tomadas por separado, esta filosofía de dividir y tratar cada parte por separado se conoce como enfoque analítico. En el análisis cuando se quiere comprender algo lo primero que se hace es dividirlo, pero no es posible 
comprender un sistema por análisis ya que pierde sus propiedades esenciales (Ackoff, 2007).

A pesar de todas las consideraciones anteriores, se puede observar cómo a las organizaciones no ha llegado ese mensaje, se sigue manejando un enfoque mecanicista y analítico y son las universidades las llamadas a mediar en esa brecha, por medio de la formación de personas que comprendan bien el concepto de sistema y lo puedan aplicar mediante el desarrollo de herramientas que se puedan poner en práctica en las organizaciones (Convenio USTA-CONTEC, 2012).

Si bien hay un enfoque y un esfuerzo hacia el manejo del concepto de sistema y del pensamiento sistémico aplicado a los sistemas de gestión y a la gestión integral en los programas del convenio, este enfoque no está estructurado ni formalizado y en consecuencia hay disparidad en su interpretación y transmisión a los estudiantes, lo cual dificulta la concepción de un modelo propio sobre el cual se puedan construir futuros desarrollos y la creación de conocimiento específico que se irradie de los programas hacia la comunidad organizacional, los consultores y las demás partes interesadas.

Russell Ackoff (2002) afirma que se está saliendo de la era de la máquina en la cual se considera al universo como una máquina creada por Dios para realizar su obra; a partir de este postulado, infiere que el hombre tenía que crear máquinas para que hicieran su trabajo, asimismo afirma que la Revolución Industrial trajo consigo la idea de mecanización, donde para entender algo, es necesario separarlo, conceptual y físicamente, en sus partes, y, además, esta revolución tiene que ver con la sustitución del hombre como fuente de trabajo, por máquinas hechas por él mismo. Es así como hombres y máquinas hacen tareas elementales y se unen para hacer el trabajo completo. En consecuencia, hay un costo al que denomina "ironía de la Revolución Industrial", en la cual se reduce el trabajo de las personas a tareas elementales que en un momento dado pudieran ser reemplazadas por máquinas.

Como antítesis, propone la era de los sistemas (Ackoff, 2002), en la que se comparte la visión, la misión y el método, considerando la organización como un sistema social mediante el pensamiento sistémico. Un sistema es más que la suma de sus partes en un todo, donde las partes actúan de manera interdependiente resaltando las cualidades de cada una para mejorar la respuesta del sistema. El modelo propuesto por Ackoff (2002) se refiere al crecimiento económico de las personas, las familias, las organizaciones y los países. Infortunadamente, se deja de lado el factor humano, la cultura, los valores, etc., y es por esto que abordar un enfoque sistémico, permite que ese crecimiento se materialice de manera gradual y efectiva para que pueda ser sostenible en el tiempo.

De otro lado, muchas organizaciones han decidido incorporar la aplicación de normas técnicas reconocidas (nacionales o internacionales). Estas normas constituyen sin duda referencias de gran utilidad que permiten dar estructura y fundamento a la manera de llevar a cabo unas determinadas actividades, y de ejercer un control sobre las mismas. La calidad, el medio ambiente y la seguridad y salud en el trabajo, son algunas de las áreas de gestión en las que fácil y rápidamente se pueden identificar normas internacionales que son ampliamente utilizadas por muchas organizaciones, aunadas a un proceso de planeación estratégica y del estudio detallado de los intereses de las partes involucradas en la gestión organizacional (gestión integral).

La gestión integral se entiende como la gestión que armoniza el medio externo con el interno para generar claridad en la conducción y gobierno de la organización, involucrando el manejo sostenible de los recursos naturales, la responsabilidad social y con las partes interesadas (Tejada y Peña, 2009). 
En consecuencia, aunando los elementos propios del pensamiento sistémico y la gestión integral, mencionados en los párrafos anteriores, se considera que este estudio beneficia en primera instancia a los estudiantes de los programas de especialización y maestría del convenio USTA-ICONTEC, porque les permitirá comprender conceptos generales sobre el pensamiento sistémico que podrán aplicar en las organizaciones de cualquier sector y en cualquier sistema de gestión, quienes como futuros egresados podrán aportar a la sostenibilidad, al mejoramiento continuo y a la gestión de las organizaciones, ya sea en calidad de docentes, consultores, auditores o asesores de sistemas de gestión.

Además, con el pensamiento sistémico se benefician las organizaciones (Ackoff, 2002), porque este contribuye a la optimización de los procesos, el cumplimiento de metas y una planeación estructurada que les permite anticiparse al entorno donde se encuentran, apoyándose en la participación de todos los integrantes de una organización (partes interesadas), quienes aportan proponiendo soluciones sistémicas, ya que tienen la visión del todo y por lo tanto la competencia para comprender las interacciones entre los procesos y proponer soluciones que beneficien al todo (sistema social).

Definir e incluir formalmente el pensamiento sistémico en los programas del convenio, permitiría la comprensión y manejo de sistemas complejos, como los que existen en cualquier organización. Utilizar esta herramienta simplificaría el entendimiento de los procesos internos y su efecto en el entorno, así como la interacción entre las partes que integran el sistema global, ayudaría a identificar algunas reglas, algunas series de patrones y sucesos para prepararnos de cara al futuro e influir sobre él en alguna medida. Requiere, además, formar parte de un programa integral de administración de la calidad, o mejor aún, de administración sistémica de la calidad, que integre los enfoques de calidad y de pensamiento sistémico brindando coherencia, armonía y coordinación a todos los subsistemas de la organización, de manera que esta última se renueve a sí misma y rediseñe su futuro constantemente. La llamada "administración sistémica”, que busca integrar la gestión integral y de pensamiento sistémico, puede acelerar el aprendizaje organizacional, tanto operativo como conceptual, más allá de las actuales capacidades de los métodos tradicionales de calidad, y, con ello, mejorar su capacidad para crear su propio futuro y promover el desarrollo -más que el crecimiento-, de las organizaciones (Ackoff, 2002).

La importancia del presente estudio radica en la necesidad de desarrollar el concepto y los elementos teóricos propuestos en el pensamiento sistémico, por cuanto en el documento maestro de la Maestría, se menciona de manera tangencial a Senge (1992), desde la necesidad de comprensión de la organización como sistema y la comprensión de las fuerzas internas y externas que impulsan el cambio y cómo son los procesos de aprendizaje por los cuales los gerentes de toda la organización llegan a entender esas fuerzas y tendencias. De igual manera, el perfil de los egresados y el impacto que tienen en la gestión organizacional, así como la generación de conocimiento y el desarrollo de competencias integrales que aporten a la inserción de las organizaciones empresariales del país en el mercado global, contribuye también al logro de bienestar que frene la inequidad social.

Bajo este contexto, la investigación buscó proponer una relación entre el pensamiento sistémico y la gestión integral para su posible incorporación en los programas de posgrado del convenio USTA-ICONTEC. Para ello, partió de la descripción de la relación teórica existente entre el pensamiento sistémico y la gestión integral. Posteriormente, dio a conocer las concepciones de pensamiento sistémico y gestión integral que tienen las distintas partes interesadas relevantes 
de los programas de posgrado del convenio USTAICONTEC. Además, se identificaron las estrategias de enseñanza-aprendizaje empleadas actualmente para el desarrollo del pensamiento sistémico en el estudiante de posgrado de sistemas de gestión, y se propusieron estrategias para el desarrollo del pensamiento sistémico en los programas de formación de posgrado del convenio USTA-ICONTEC.

\section{METODOLOGÍA}

El proceso metodológico realizado para dar cumplimiento a la investigación propuesta incluye el paradigma, el enfoque, el tipo de investigación, el método y los criterios de validez y confiabilidad. En la Figura 1 se precisan los aspectos mencionados y se amplían a continuación.
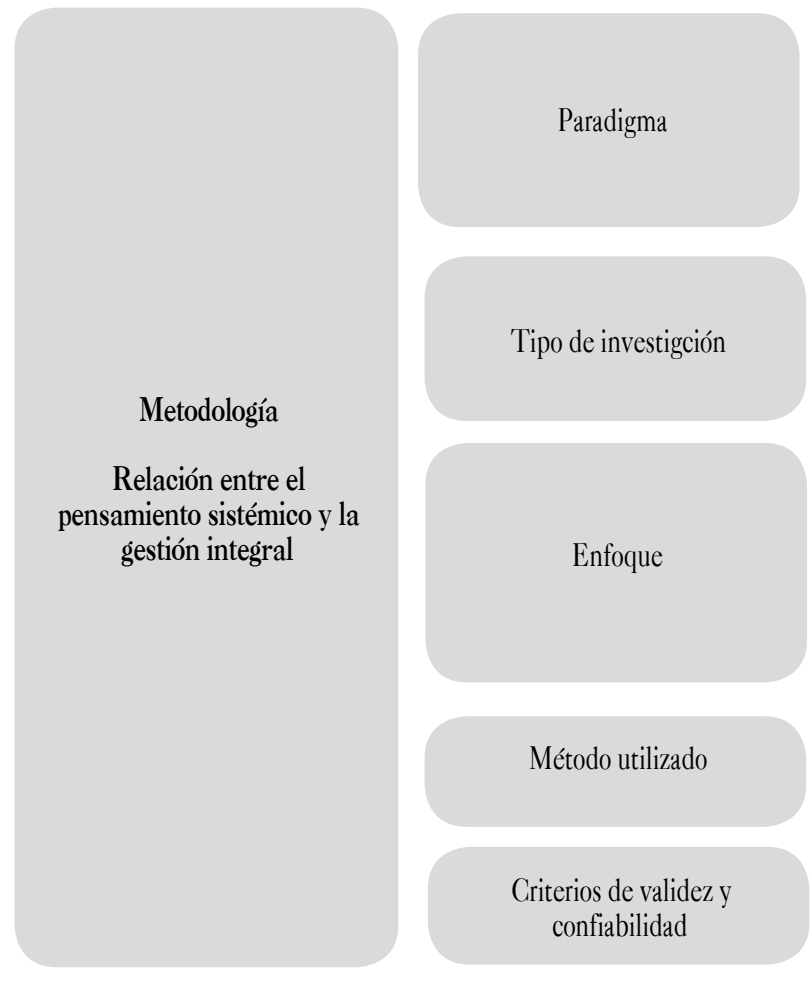

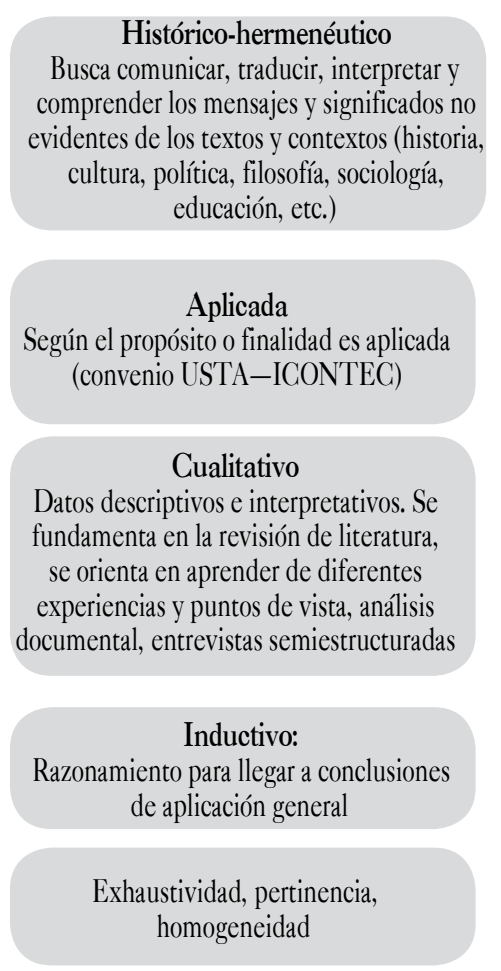

Fuente: elaboración propia (2016).

Figura 1. Metodología de la investigación.

La investigación se realizó bajo el paradigma histórico hermenéutico. Se desarrolló en los programas de posgrado del convenio USTA-ICONTEC, caracterizado por la aplicación, utilización y consecuencias prácticas de los conocimientos donde se indaga conocer y proponer un modelo que permita tomar decisiones, modificar procedimientos, normatividad y construir propuestas, si así se concluye en la investigación (Lara, 2011, p. 52). Corresponde a una investigación cualitativa, debido a que se fundamenta en la revisión de literatu- ra, se orienta en aprender de diferentes experiencias y puntos de vista, de procesos, de documentos y estudios realizados. Se aplica el método inductivo en el cual se utiliza el razonamiento para obtener conclusiones que parten de hechos particulares aceptados como válidos, para llegar a conclusiones cuya aplicación sea de carácter general (Hernández, Fernández y Baptista, 2014).

La confiabilidad está dada por la precisión en los mecanismos de recolección de información. A continuación, los criterios de validez y confiabilidad, de acuer- 
do con Hernández, Fernández y Baptista (2014), son la exhaustividad, la homogeneidad y la pertinencia.

Como técnica de recolección se tiene en cuenta el análisis de contenido. Este se define como una técnica de investigación que identifica y describe de una manera objetiva y sistemática las propiedades de un texto con la finalidad de obtener conclusiones; adicionalmente, permite de manera objetiva y sistemática la interpretación de contenidos (Hernández, Fernández y Baptista, 2014).

Se realizó una revisión sistemática exploratoria siguiendo la metodología de Manchado (2009) de artículos publicados en las bases de datos Science Direct, Ebscohost, Jstor y SciVerse Scopus, entre 2005 y 2015, usando términos MeSH pensamiento sistémico y gestión y las ecuaciones de búsqueda pensamiento sistémico y gestión. Los artículos son seleccionados de acuerdo con los siguientes criterios de inclusión: artículos relacionados con aplicación de pensamiento sistémico en sistemas de gestión de calidad, ambiental, salud en el trabajo y riesgos, entre otros. Una vez identificados los textos, estos son organizados en una matriz documental con el fin de identificar documentos repetidos. Posteriormente, a través del análisis de contenido se revisan uno a uno los textos completos y los resultados del análisis de cada una de las categorías son organizados haciendo uso de una matriz analítica. Las categorías analizadas son: campo de aplicación, concepto de pensamiento sistémico, elementos del pensamiento sistémico, teoría abordada del pensamiento sistémico, conceptos de gestión, elementos de la gestión, teoría abordada de la gestión, conceptos de gestión integral, elementos de la gestión integral y teoría abordada de la gestión integral.

En la figura 2 se presenta el flujograma de depuración de los artículos.

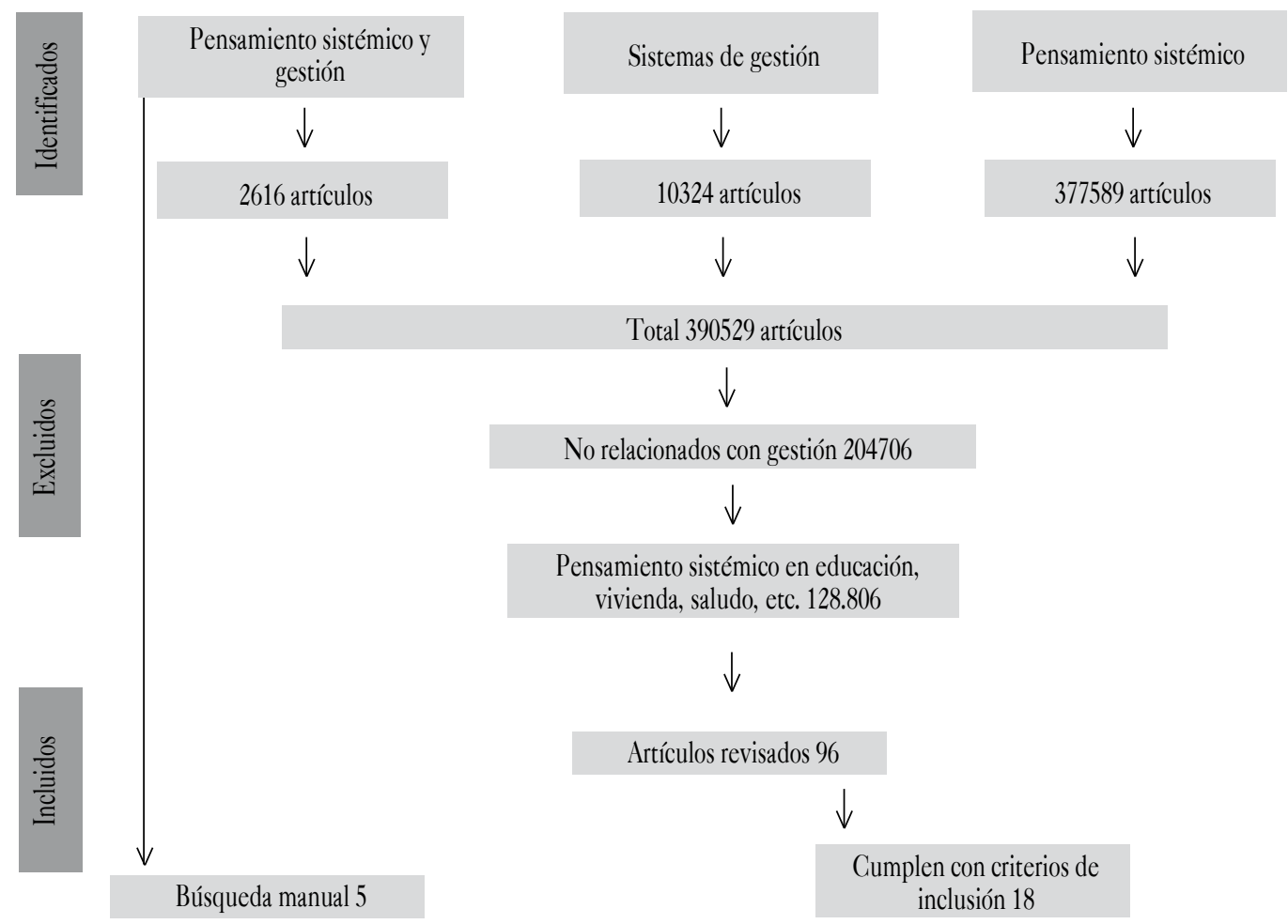

Fuente: elaboración propia (2016).

Figura 2. Diagrama selección de artículos 


\section{Proceso metodológico para determinar las concepciones de pensamiento sistémico y gestión integral de las partes interesadas relevantes de los programas de posgrado del convenio USTA- ICONTEC}

Es preciso aclarar que si bien hay otras partes interesadas, como empleadores y directivos, las que se seleccionaron son las partes interesadas relevantes, porque son las que directamente están inmersas en el proceso de formación, el cual es el que se pretende intervenir con la investigación. Para conocer las concepciones de pensamiento sistémico y gestión integral de las partes interesadas (relevantes) del convenio, acorde con el enfoque cualitativo, se define una propuesta de entrevista semiestructurada, con el objetivo de conocer en qué medida han apropiado los docentes, egresados y estudiantes de los posgrados del convenio USTA-ICONTEC, los conceptos de pensamiento sistémico, gestión integral y gestión integrada.

De acuerdo con las categorías definidas (pensamiento sistémico, gestión integral y gestión integrada), el instrumento se formula en primera instancia con siete preguntas, 5 abiertas y 2 con opción de selección; y luego se somete a revisión por expertos con el fin validar la entrevista semiestructurada bajo los criterios de validez, objetividad, claridad y pertinencia.

El grupo de expertos generó recomendaciones en cuanto al contenido del instrumento, las cuales se tienen en cuenta y el instrumento se formula quedando estructurado con 8 preguntas: cuatro abiertas (1, 5, 6 y 7), 2 cerradas (pregunta 2 con 5 alternativas, pregunta 4 con 3 alternativas de respuesta) y 2 preguntas dicotómicas (3 y 8). Es preciso aclarar que la pregunta 3 se enfoca de manera diferente para cada grupo de entrevistados (docentes, egresados y estudiantes).

\section{Método para identificar las estrategias de enseñanza-aprendizaje}

Para el desarrollo de este objetivo, se tuvieron en cuenta los contenidos de los programas de posgrado y los proyectos de investigación de la línea de gestión integral desarrollados desde la aprobación de la Maestría. Inicialmente se hace un análisis de los documentos maestros de los programas de posgrado del convenio USTA-ICONTEC: Especialización en Administración y Gerencia de Sistemas de Calidad (2014a) y Maestría en Calidad y Gestión Integral (2014b), precisando los elementos diferenciadores y las características propias de cada uno de ellos.

Posteriormente, se realiza el análisis de contenido de cada una de las fichas técnicas del programa de especialización. Los elementos analizados fueron: módulo, dimensión, semestre, créditos, objetivos y las categorías propias del presente estudio como son pensamiento sistémico, sistemas de gestión y gestión integral. Para el caso de la Maestría, se revisa el módulo, la dimensión, los objetivos, las competencias a desarrollar además de las categorías propias del presente estudio.

Para finalizar, se realiza un análisis de los proyectos de investigación de la Maestría del convenio. Del total de trabajos realizados en las diferentes líneas de investigación, desde el año 2008 hasta septiembre de 2015, 38 corresponden a línea de investigación de gestión integral, de los cuales se analizaron 25. Se aborda el título, los autores, el director, el año y, por supuesto, las categorías del estudio (pensamiento sistémico, gestión integral y gestión integrada). Seguidamente, a través del análisis de contenido, se revisan los textos en su totalidad y se sintetizan en una matriz analítica.

En la Figura 3 se presenta el flujograma de depuración de los proyectos. 


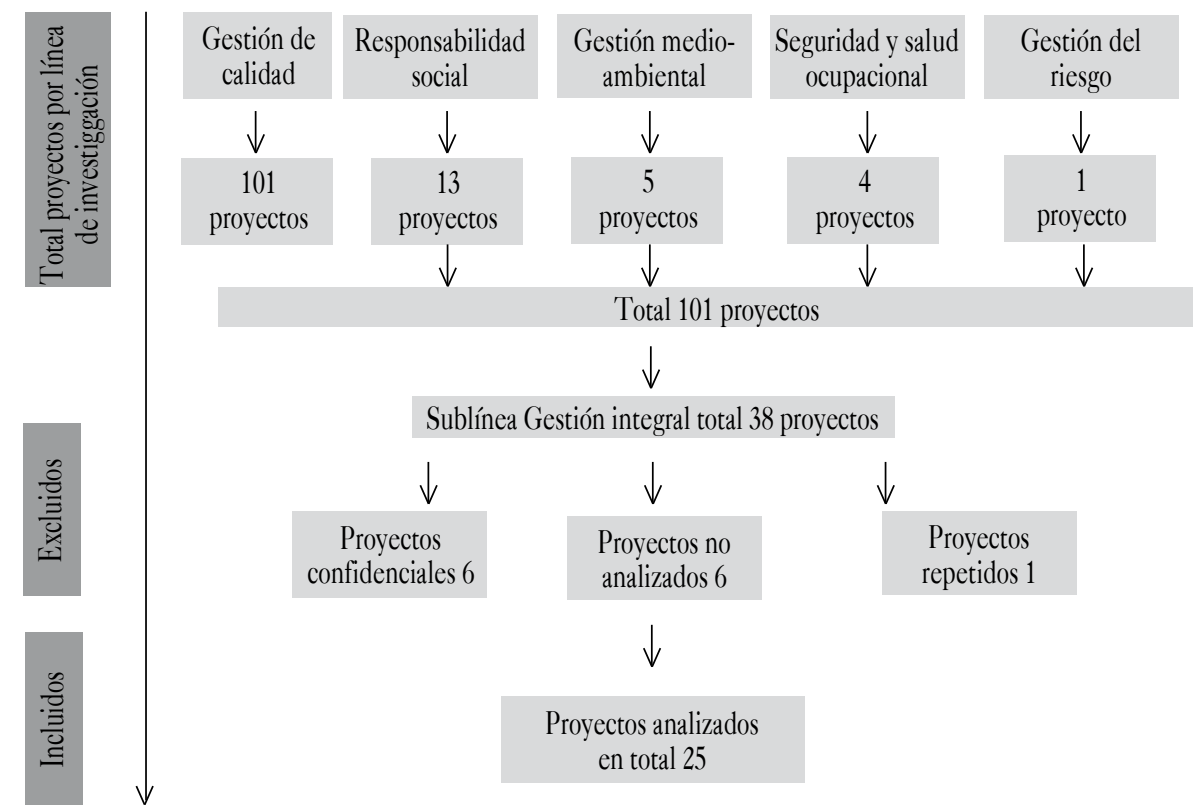

Fuente: elaboración propia (2016).

Figura 3. Diagrama de selección de proyectos de investigación

\section{Metodología para la propuesta de estrategias para el desarrollo del pensamiento sistémico en los progiramas de formación de posǵrado del convenio USTA-ICONTEC}

Para llegar a la propuesta para el desarrollo del pensamiento sistémico en los posgrados del convenio, se aplica la triangulación de métodos de recolección de datos, entendida como el uso de diferentes métodos de investigación y un análisis de sus conclusiones a fin de obtener una idea más completa del fenómeno que se investiga. Se aplica la triangulación intrametodológica, es decir, dentro del mismo método cualitativo (Hernández, Fernández y Baptista, 2014), con herramientas como análisis de contenido, entrevista semiestructurada y revisión sistemática. Se analizan las conclusiones de cada uno de los tres primeros objetivos con el fin de presentar una propuesta de ficha técnica para el módulo de pensamiento sistémico que incluye información general, descripción del módulo, objetivos, competencias desarrolladas, saberes esenciales, estrategias metodológicas y bibliografía sugerida.

\section{RESULTADOS Y DISCUSIÓN}

\section{Fundamentación y relación existente entre el pensamiento sistémico y la gestión inteǵral}

\section{Pensamiento sistémico}

Un sistema se define como un conjunto de dos o más elementos, cuando se fragmenta pierde sus propiedades esenciales y por ende no puede entenderse por análisis; pero, ¿̇cuál podría considerarse una definición acertada de pensamiento sistémico?, con el ánimo de precisarla, en la Tabla 1 se presentan las definiciones y elementos relacionados con pensamiento sistémico, desde la visión de diferentes autores. 
Tabla 1. Conceptos del pensamiento sistémico.

\begin{abstract}
Autor
Concepto

Un sistema es más que la suma de sus partes. Las partes propias de un sistema pueden representar otros sistemas y cada sistema

Russell por sí mismo puede ser parte de otro mayor. El pensamiento sistémico tiende a ver las cosas como partes de un todo mayor, más

Ackoff que como un todo que se descompone. Las organizaciones son sistemas que contienen elementos intencionados que tienen un propósito común, sistema social (Ackoff, 2002). El pensamiento complejo se reconoce como un pensamiento que relaciona y complementa. Su objeto y sujeto de estudio es el
todo, a través de sus efectos, defectos, dinamismo y estática, reconociendo la interrelación del todo con sus partes y viceversa,
dentro de un entramado. Lo complejo designa hoy una comprensión del mundo como entidad donde todo se encuentra entre-
lazado, como en un tejido compuesto de finos hilos (Morín, 2015).

Un modo de analizar -y un lenguaje para describir y comprender- las fuerzas e interrelaciones que modelan el comportamiento

Peter Senge de los sistemas. Esta disciplina nos permite cambiar los sistemas con mayor eficacia y actuar en forma más acorde con los procesos del mundo natural y económico (Senge, 1999).

Joseph El pensamiento sistémico es un método de identificar algunas reglas, algunas series de patrones y sucesos para prepararnos de O'Connor cara al futuro e influir sobre él de alguna medida, nos aporta cierto control. El pensamiento sistémico contempla el todo y las partes, así como las conexiones entre las partes, y estudia el todo para poder comprender las partes. Es lo opuesto al reduccio-

Ian McDer- nismo, es decir, la idea de que algo es simplemente la suma de sus partes. Una serie de partes que no están conectadas no es un mott sistema, es sencillamente un montón (O’Connor y McDermott, 1998).

Fritjot Capra sistema es diseccionado, ya sea física o teóricamente, en elementos aislados. Si bien podemos discernir partes individuales en

Según la visión sistémica, las propiedades esenciales de un organismo o sistema viviente, son propiedades del todo que ninguna de las partes posee. Emergen de las interacciones y relaciones entre las partes. Estas propiedades son destruidas cuando el todo sistema, estas partes no están aisladas y la naturaleza del conjunto es siempre distinta de la mera suma de sus partes (Capra, 1998, p. 48).
\end{abstract}

Fuente: elaboración propia (2016).

El presente estudio se desarrolló al interior del convenio USTA-ICONTEC, involucrando a sus diferentes actores (docentes, egresados, estudiantes) quienes desde sus trabajos pueden aportar en la mejora continua de las organizaciones, en sus procesos y en los sistemas de gestión normalizados, razón por la que se reconoce el concepto de pensamiento sistémico formulado por Ackoff (2002), que complementa la definición propuesta con las siguientes consideraciones: la síntesis o poner juntos los elementos es la clave del pensamiento sistémico, un pensador de la era de la máquina (analítico) al desarrollar un estudio separaría las partes hasta su mínima expresión, mientras que un pensador sistémico al realizar la misma tarea, haría un estudio del sistema inicial, seguido del sistema que lo contenga y del sistema social que aplica para el estudio en cuestión.

De manera complementaria a las definiciones, se hace un compendio de los principios o propiedades del pensamiento sistémico, además de las características de cada uno de ellos. Estos se precisan en la Tabla 2. 
Tabla 2. Principios/propiedades del pensamiento sistémico.

\begin{tabular}{|c|c|c|c|}
\hline Autor & & Principios/propiedades & Características \\
\hline \multirow{3}{*}{$\begin{array}{l}\text { Russell } \\
\text { Ackoff }\end{array}$} & 1 & $\begin{array}{l}\text { Identificar un todo contenedor (sis- } \\
\text { tema) del cual el objeto por explicar } \\
\text { es una parte. }\end{array}$ & $\begin{array}{l}\text { Las propiedades de cada elemento del conjunto tienen las propiedades o el com- } \\
\text { portamiento del conjunto, tomando un todo. }\end{array}$ \\
\hline & 2 & $\begin{array}{l}\text { Explicar el comportamiento o pro- } \\
\text { piedades del todo contenedor. }\end{array}$ & $\begin{array}{l}\text { Las propiedades o comportamientos de cada elemento y la forma en que afectan el } \\
\text { todo dependen de las propiedades y comportamiento de al menos otro elemento } \\
\text { en el conjunto. }\end{array}$ \\
\hline & 3 & $\begin{array}{l}\text { Explicar entonces el comporta- } \\
\text { miento o las propiedades del objeto } \\
\text { por explicar en términos de su(s) } \\
\text { papel(es) o función(es) dentro de su } \\
\text { todo contenedor. }\end{array}$ & $\begin{array}{l}\text { Cada subgrupo posible de elementos del conjunto tiene las dos primeras propieda- } \\
\text { des; cada una tiene un efecto no independiente en el total; en consecuencia, no se } \\
\text { puede descomponer el total en subconjuntos independientes. }\end{array}$ \\
\hline \multirow{7}{*}{ Edgar Morin } & 1 & Principio sistemático u organizativo. & $\begin{array}{l}\text { La unión del conocimiento de las partes con el conocimiento del todo, cuyo objeti- } \\
\text { vo consiste en combatir la idea reduccionista de que el todo es tan solo la suma de } \\
\text { las partes e incorporar la importancia de las interconexiones. }\end{array}$ \\
\hline & 2 & Principio holográmico. & $\begin{array}{l}\text { Representa una realidad que contiene la totalidad de la información, por lo cual } \\
\text { permite un análisis desde todos los ángulos. Este principio complementa el holis- } \\
\text { mo (el todo) y el reduccionismo (las partes). }\end{array}$ \\
\hline & 3 & $\begin{array}{l}\text { Principio del bucle retroactivo } 0 \\
\text { realimentación. }\end{array}$ & $\begin{array}{l}\text { El efecto se vuelve causa, la causa se vuelve efecto, los productos son productores, } \\
\text { el individuo hace cultura y la cultura hace a los individuos. Permite la autonomía } \\
\text { organizacional del sistema y rompe la causalidad lineal. }\end{array}$ \\
\hline & 4 & $\begin{array}{l}\text { Principio de } \\
\text { autonomía/dependencia. }\end{array}$ & $\begin{array}{l}\text { La cultura y el medio ambiente tienen influencia de manera directa en el desarro- } \\
\text { llo de la autonomía de los seres vivos. Para mantener su autonomía, toda organi- } \\
\text { zación necesita de la apertura al ecosistema del que se nutre y al que transforma. }\end{array}$ \\
\hline & 5 & $\begin{array}{l}\text { Principio de recursividad } \\
\text { organizacional. }\end{array}$ & $\begin{array}{l}\text { Consiste en un "bucle generador" a través del cual los efectos o productos al mis- } \\
\text { mo tiempo son causantes y productores del proceso mismo, donde los estados fina- } \\
\text { les son necesarios para la generación de los estados iniciales. }\end{array}$ \\
\hline & 6 & Principio dialógico. & $\begin{array}{l}\text { Permite asumir la inseparabilidad de nociones contradictorias para concebir un fe- } \\
\text { nómeno. No asume la separación de los contrarios, sino que lo dos términos coexis- } \\
\text { ten sin dejar de ser antagónicos, y nos permite la comprensión del uno por el otro. }\end{array}$ \\
\hline & 7 & $\begin{array}{l}\text { Principio de reintroducción del que } \\
\text { conoce en todo conocimiento. }\end{array}$ & $\begin{array}{l}\text { Todo conocimiento es una reconstrucción, una traducción que una mente o cere- } \\
\text { bro hace en una cultura y un tiempo determinados. El sujeto construye su realidad } \\
\text { desde su condición biológica, espiritual y social, incluyendo lo que otros hicieron } \\
\text { antes. }\end{array}$ \\
\hline
\end{tabular}




\begin{tabular}{|c|c|c|c|}
\hline Autor & & Principios/propiedades & Características \\
\hline \multirow{4}{*}{ Peter Senge } & 1 & $\begin{array}{l}\text { No se puede dividir el elefante } \\
\text { en dos. }\end{array}$ & $\begin{array}{l}\text { La comprensión de la mayoría de los problemas requiere ver la totalidad del siste- } \\
\text { ma que genera dichos problemas. No de manera independiente, muchos proble- } \\
\text { mas se encuentran en las interacciones. }\end{array}$ \\
\hline & 2 & $\begin{array}{l}\text { Las causas y efectos no están estre- } \\
\text { chamente relacionados en el tiempo } \\
\text { y el espacio. }\end{array}$ & $\begin{array}{l}\text { No actúe cerca del síntoma del problema, retroceda en el tiempo para eliminar } \\
\text { la raíz. }\end{array}$ \\
\hline & 3 & $\begin{array}{l}\text { Es posible tenerlo todo pero no al } \\
\text { mismo tiempo. }\end{array}$ & $\begin{array}{l}\text { Al proponer soluciones sistémicas, tenga en cuenta las inevitables demoras tempo- } \\
\text { rales. Las demoras temporales y otros aspectos sutiles del sistema solo se eviden- } \\
\text { cian con el tiempo y la experimentación. }\end{array}$ \\
\hline & 4 & Las salidas fáciles no son salidas. & $\begin{array}{l}\text { Todos se sienten cómodos aplicando soluciones típicas a los problemas, atenién- } \\
\text { dose a lo conocido. Si la solución fuera obvia, tal vez ya se hubiera encontrado. Se } \\
\text { insiste en soluciones conocidas cuando los problemas empeoran. }\end{array}$ \\
\hline \multirow{4}{*}{$\begin{array}{l}\text { Joseph } \\
\text { O'Connor }\end{array}$} & 5 & $\begin{array}{l}\text { La conducta empeora antes de } \\
\text { mejorar. }\end{array}$ & $\begin{array}{l}\text { La realimentación compensadora siempre implica una "demora", un paréntesis } \\
\text { entre el beneficio a corto plazo y el perjuicio a largo plazo, con lo cual se pueden } \\
\text { presentar momentos de desesperación entre los miembros del equipo de trabajo. }\end{array}$ \\
\hline & 1 & $\begin{array}{l}\text { Partes interconectadas que funcio- } \\
\text { nan como un todo. La disposición de } \\
\text { las piezas es fundamental. }\end{array}$ & $\begin{array}{l}\text { Sistemas formados por partes distintas y con funciones diferentes pueden estar } \\
\text { organizados en torno a las mismas reglas generales. }\end{array}$ \\
\hline & 2 & Cambia si se quitan o añaden piezas. & $\begin{array}{l}\text { Si se divide un sistema en dos, no se consiguen dos sistemas más pequeños, sino } \\
\text { un sistema defectuoso que probablemente no funcionará. }\end{array}$ \\
\hline & 3 & $\begin{array}{l}\text { Las partes están conectadas y fun- } \\
\text { cionan todas juntas. }\end{array}$ & $\begin{array}{l}\text { El comportamiento de un sistema dependerá de cómo se conecten las partes, más } \\
\text { que de cuáles sean esas partes. }\end{array}$ \\
\hline
\end{tabular}

McDermott

4 Su comportamiento depende de la estructura global.
Si se cambia la estructura, se modifica el comportamiento del sistema.

\begin{tabular}{|c|c|l|l} 
& 1 & El cambio de las partes al todo. & $\begin{array}{l}\text { Los sistemas vivos son totalidades integradas, cuyas propiedades no pueden ser } \\
\text { reducidas a las de sus partes más pequeñas. Sus propiedades esenciales son del } \\
\text { conjunto, que ninguna de las partes tiene por sí sola. }\end{array}$ \\
Fritjot Capra & $\begin{array}{l}\text { La habilidad para focalizar la aten- } \\
\text { ción alternativamente en distintos } \\
\text { niveles sistémicos. }\end{array}$ & $\begin{array}{l}\text { A distintos niveles sistémicos corresponden distintos niveles de complejidad. En } \\
\text { cada nivel los fenómenos poseen propiedades que no se dan en niveles inferiores. }\end{array}$ \\
& $3 \quad$ No hay partes en absoluto. & $\begin{array}{l}\text { Lo que denominamos parte, es meramente un patrón dentro de una inseparable } \\
\text { red de relaciones. }\end{array}$
\end{tabular}

Fuente: elaboración propia (2016).

La Tabla 2 refiere los principios/propiedades del pensamiento sistémico. Una vez analizada se establecen las diferencias y similitudes desde diversos autores, lo que se presenta a continuación en la Tabla 3. 
Tabla 3. Principios del pensamiento sistémico desde diversos autores.

\begin{tabular}{|c|c|c|c|c|c|}
\hline Principio & Russel Ackoff & Edgar Morin & Peter Senge & $\begin{array}{c}\text { Joseph O’ Connor - Ian Mc- } \\
\text { Dermott }\end{array}$ & Fritjot Capra \\
\hline 1 & $\begin{array}{l}\text { Identificar un todo conte- } \\
\text { nedor (sistema) del cual } \\
\text { el objeto por explicar es } \\
\text { una parte. }\end{array}$ & $\begin{array}{l}\text { Principio sistemático } \\
\text { u organizativo. }\end{array}$ & $\begin{array}{l}\text { No se puede dividir } \\
\text { el elefante en dos. }\end{array}$ & $\begin{array}{l}\text { Partes interconectadas que } \\
\text { funcionan como un todo. La } \\
\text { disposición de las piezas es } \\
\text { fundamental. }\end{array}$ & $\begin{array}{l}\text { El cambio de las } \\
\text { partes al todo. }\end{array}$ \\
\hline 2 & $\begin{array}{l}\text { Explicar el comporta- } \\
\text { miento o propiedades del } \\
\text { todo contenedor. }\end{array}$ & $\begin{array}{l}\text { Principio } \\
\text { holográmico. }\end{array}$ & & $\begin{array}{l}\text { Cambia si se quitan o añaden } \\
\text { piezas. }\end{array}$ & $\begin{array}{l}\text { No hay partes en } \\
\text { absoluto. }\end{array}$ \\
\hline 3 & $\begin{array}{l}\text { Explicar entonces el } \\
\text { comportamiento o las } \\
\text { propiedades del objeto } \\
\text { por explicar en términos } \\
\text { de su(s) papel(es) o } \\
\text { función(es) dentro de su } \\
\text { todo contenedor. }\end{array}$ & $\begin{array}{l}\text { Principio de autono- } \\
\text { mía/dependencia. }\end{array}$ & & $\begin{array}{l}\text { Las partes están conectadas y } \\
\text { funcionan todas juntas. }\end{array}$ & \\
\hline 4 & & $\begin{array}{l}\text { Principio del bucle } \\
\text { retroactivo o reali- } \\
\text { mentación. }\end{array}$ & & $\begin{array}{l}\text { Su comportamiento depende de } \\
\text { la estructura global. }\end{array}$ & $\begin{array}{l}\text { La habilidad para } \\
\text { focalizar la atención } \\
\text { alternativamente } \\
\text { en distintos niveles } \\
\text { sistémicos. }\end{array}$ \\
\hline 5 & & $\begin{array}{l}\text { Principio de recursi- } \\
\text { vidad organizacional. }\end{array}$ & $\begin{array}{l}\text { Las causas y efectos } \\
\text { no están estrecha- } \\
\text { mente relacionadas. } \\
\text { Con el tiempo y el } \\
\text { espacio. }\end{array}$ & & \\
\hline 6 & & Principio dialógico. & & & \\
\hline 7 & & $\begin{array}{l}\text { Principio de reinduc- } \\
\text { ción del que conoce } \\
\text { en todo conoci- } \\
\text { miento. }\end{array}$ & & & \\
\hline
\end{tabular}

Fuente: elaboración propia (2016).

Se observa que el principio identificar un todo contenedor es mencionado por todos los autores con diferente terminología, pues hace referencia a las partes y al todo así como a las interconexiones que existen en un sistema. El principio explicar el comportamiento del todo contenedor, representa la totalidad de la información, se puede observar la similitud que guarda con el holográmico propuesto por Morin, asimismo O'Connor y Mc-
Dermott afirman que un sistema cambia si se quitan 0 añaden piezas y Capra, al referirse que no hay partes en absoluto. El principio el comportamiento de objeto en función del todo contenedor (Ackoff, 2002), también es similar al principio de autonomíaldependencia y al principio de las partes están conectadas y funcionan todas juntas (O'Connor y McDermott, 1998). En el principio de bucle retroactivo, su comportamiento depende de la estructura 
global, la habilidad para focalizar la atención alternativamente en distintos niveles sistémicos.

El principio de recursividad organiæacional tiene cierta similitud con el propuesto por Senge (1992). Las causas y efectos no están estrechamente relacionados con el tiempo y el espacio. En los principios dialógico y de reinducción, no se observa una relación directa, al igual que en los demás principios propuestos por este autor. Para efectos del presente estudio se aplicarán los principios del pensamiento sistémico de Ackoff (2002), dada su amplitud y la relación que puede observarse con los principios propuestos por los demás autores.

Los anteriores elementos pueden resultar totalmente teóricos si no se contrastan con los sistemas de gestión normalizados, por lo cual no es fortuito precisar que desde su denominación o su génesis, los sistemas de gestión normalizados se diseñaron precisamente como sistemas, en consecuencia, tienen estrecha relación con los elementos y principios de pensamiento sistémico. En concordancia con lo anterior, la norma ISO 9000:2005 define ocho principios de gestión de calidad dentro de los cuales se encuentra el enfoque de sistema para la gestión y el enfoque basado en procesos.

En cuanto al enfoque de sistema para la gestión, la mencionada norma precisa que "identificar, entender y gestionar los procesos interrelacionados como un sistema, contribuye a la eficacia y eficiencia de una organización en el logro de sus objetivos" (ICONTEC, 2005). Mientras que el enfoque basado en procesos, hace referencia a que un resultado deseado se alcanza más eficientemente cuando las actividades y los recursos se gestionan como un proceso, y hace énfasis en la importancia de "la identificación y gestión sistemática de los procesos empleados en la organización y en particular las interacciones entre tales procesos", también se afirma que para que las organizaciones funcionen de manera eficaz, tienen que identificar y gestionar numerosos procesos interrelacionados y que interactúan (ISO 9001, 2005).
Todas las normas ISO poseen requisitos comunes y adoptaron el ciclo de mejora continua PHVA, no obstante, muchas contienen requisitos similares de manera distinta, por ello en algunas se incluye un anexo con los numerales que tienen relación entre sí. Con el fin de evitar los problemas de comprensión generados, la ISO desarrolla el Anexo SL, que describe el contexto para un sistema de gestión genérico, y lo formula en tres secciones: 1) estructura de alto nivel, 2) texto base idéntico y, 3) términos y definiciones comunes. La ISO 9001:2015 y la ISO 14001:2015 ya se exponen en este contexto y a partir de allí todas las normas técnicas revisadas deberán incluirlo (ISO, 2015). La unificación de la estructura genera beneficios para las empresas en la implementación, para los auditores, para los consultores, además reduce costos y facilita la integración de sistemas de gestión normalizados.

\section{Gestión integral}

La palabra gestión viene del latín gestio,-ōnis, que significa acción y efecto de gestionar o acción y efecto de administrar. Al referirse a organizaciones se considera el concepto de gestión de negocios, definido como cuasicontrato que se origina por el cuidado de intereses ajenos sin mandato de su dueño. Gestionar es probablemente el verbo que más se utiliza en el lenguaje empresarial, significa: "Hacer diligencias conducentes al logro de un negocio o de un deseo cualquiera, es hacer todo para que las cosas se pongan en marcha y funcionen. Y eso vale para cualquier área de una organización”. (Real Academia Española, 2014).

De igual modo, se hace referencia a la definición de integral (del latín mediev.integralis), que comprende todos los elementos o aspectos de algo, adicionalmente, dicho de cada una de las partes de un todo: que entra en su composición sin serle esencial, de manera que el todo puede subsistir, aunque incompleto, sin ella.

Desde el enfoque de la Maestría de Calidad y Gestión Integral del convenio USTA-ICONTEC, Te- 
jada y Peña (2009) concluyen que la dinámica de los mercados y de la globalización hace que hoy en día las organizaciones sean consideradas como sistemas, generando nuevos desafíos para los administradores, por cuanto además de los procesos internos, deben incluir el entorno como una variable que determina la orientación del sistema productivo. La organización como un sistema abierto debe desarrollar su capacidad para alcanzar resultados planificados de operaciones internas así como en el entorno de la empresa. En conclusión, la gestión integral involucra y armoniza el medio externo con el interno para generar claridad en la conducción y gobierno de la organización, involucrando además, el manejo adecuado y sostenible de los recursos ambientales, la responsabilidad con la sociedad y con las partes interesadas.

Por lo anterior, los programas de especialización del convenio USTA-ICONTEG tienen el desafío de propiciar mayor nivel competitivo para las organizaciones colombianas y latinoamericanas por medio de la reflexión, la propuesta de modelos y teorías y la diseminación en el campo de la gestión integral y, en efecto, esta definición se reconoce para el desarrollo del proyecto del presente proyecto de investigación.

\section{Gestión integrada}

En el diccionario de la Real Academia Española (2014) no se incluye la palabra integrada, registra "integrar" que viene del latín integrāare, como sinónimo de renovar y completar, que significa: constituir un todo. Completar un todo con las partes que faltaban. Hacer que alguien 0 algo pase a formar parte de un todo.

La gestión integrada es una forma de enfocar las actividades de una organización para gestionar integralmente las variables calidad, ambiente, seguridad y salud ocupacional y responsabilidad social corporativa, teniendo como propósito el logro de una política integrada de gestión. Es una manera de responder a las nuevas exigencias técnicas en los mercados nacionales e internacionales.

Para Karapetrovic (2003), la gestión integrada en considerada como el conjunto de procesos interconectados que comparten un conjunto de recursos humanos, información, materiales, infraestructuras y recursos financieros, con el fin de lograr un conjunto de objetivos relacionados con la satisfacción de una gran variedad de stakeholders. Karapetorovic considera que la integración de los sistemas de gestión se puede definir como "la implementación de los diferentes sistemas de gestión de función específica en un único y más eficaz sistema de gestión integrado". Para efectos del presente estudio se asume la definición de sistema integrado de gestión propuesta por Karapetrovic y Willborn (1998), como un conjunto de procesos interconectados que comparten un conjunto de recursos humanos, información, materiales, infraestructuras y recursos financieros, con el fin de lograr un conjunto de objetivos relacionados con la satisfacción de una gran variedad de partes interesadas.

\section{Relación entre pensamiento sistémico y sistemas de gestión}

El concepto y los patrones conceptuales de pensamiento sistémico se estudian por diferentes investigadores en áreas como gestión de proyectos, seguridad y salud en el trabajo y gestión ambiental en sectores públicos y privados. En los documentos se afirma que existe relación entre el pensamiento sistémico y los sistemas de gestión. En disciplinas como gestión de proyectos, representa la posibilidad de minimizar el riesgo, también, permite reducir la incertidumbre entre la teoría y la práctica; en seguridad y salud en el trabajo, la cultura de prevención aunada al pensamiento sistémico mejora el desempeño del sistema; en materia ambiental el concepto se hace más tangible por cuanto está estrechamente relacionado con el desarrollo sostenible, considerando relaciones en vez de cosas y es aplicable 
a sistemas urbanos, regionales, económicos y sociales, entre otros.

\section{Integración de los sistemas de gestión}

Utilizando la ecuación de búsqueda Integration of management systems, en artículos publicados desde el año 2005 y hasta el 2015, en las bases de datos Science Direct, Ebscohost,Jstore y SciVerse Scopus se encuentran 30 artículos de los cuales 18 cumplen con las variables y por lo tanto se analizan. La matriz bibliométrica se diseña bajo los siguientes parámetros: título original (en inglés, portugués o español), referencia (corresponde al título en español), el tipo de documento, los autores, el país, el año, el campo de aplicación, categorías pensamiento sistémico, sistemas de gestión y gestión integral, subcategoría (concepto, elementos y teoría abordada) seguido de la descripción de la teoría, resultados significativos y dirección electrónica del documento. Algunos estudios se refieren a la integración de sistemas de gestión normalizados, otros se fundamentan en ventajas de la integración, metodologías, niveles de integración y auditorías, entre otros.

Considerando la tesis de Bernardo y las diferentes estrategias de integración se realiza el análisis de los 18 artículos relacionados, definiendo la estrategia y la metodología, se precisa: 1) las definiciones de sistema integrado de gestión desde la visión de diferentes autores como Karapetrovic, Abad (2014) y Bernardo, entre otros; 2) un análisis detallado de las estrategias de integración y las alternativas de implementación desde la visión de diferentes autores; 3 ) se analiza el nivel de integración de los sistemas de gestión planteado por Karapetrovic; 4) las estrategias y metodologías desarrolladas por organismos de normalización que han publicado guías para la integración de sistemas de gestión, tales como: AS / NZS 4581:1999 en Australia y Nueva Zelanda; AS/NZS: 1999, DS 8001:2005 en Dinamarca: DS:2005, en España; UNE 66177:2005, AENOR:2005 y PAS 99:2012 en el Reino Unido; 5) análisis de la integración de las audi- torías y, 6) la responsabilidad social corporativa como eje de integración con otros sistemas normalizados.

A partir de las consideraciones realizadas producto del análisis de los artículos mencionados, se concluye que existe una relación directa entre el pensamiento sistémico y la gestión integral, conclusión que se sustenta en que un sistema es más que la suma de sus partes. Las partes propias de un sistema pueden representar otros sistemas y cada sistema por sí mismo puede ser parte de otro mayor. El pensamiento sistémico tiende a ver las cosas como partes de un todo mayor, más que como un todo que se descompone. Ackoff (2002) aborda el concepto de pensamiento sistémico en las organizaciones, las cuales considera como sistemas que contienen elementos intencionados que tienen un propósito común, sistema social. De otra parte, la gestión integral incluye elementos internos (la cultura organizacional, la estrategia y la gestión integrada), como elementos externos (desarrollo social, protección ambiental y crecimiento económico).

\section{Concepciones de pensamiento sistémico y gestión integral que tienen las distintas partes interesadas relevantes de los programas de posǵrado del convenio USTA-ICONTEC}

Para el cumplimiento de este objetivo se realizan en total 13 entrevistas semiestructuradas, de las cuales corresponden tres (3) a docentes, cinco (5) a egresados y cinco (5) a estudiantes, se digitan en formulario de Google® y se compilan en Excel. Posteriormente, se realiza el análisis cualitativo en la aplicación ATLAS.ti. Basados en una herramienta de la teoría fundamentada llamada microanálisis, se realiza la codificación general de todas las respuestas, 104 en total. Cuando se llega al punto de saturación para identificar la relación entre pensamiento sistémico y gestión integral, no se realizan más entrevistas. 
Las respuestas se analizan a partir de las redes que genera la aplicación ATLAS.ti, a continuación se interpretan por pregunta y temas de mayor relevancia. Cabe anotar que hay una gran variedad de respuestas, en muchos casos la misma con diferentes palabras.

\section{Pregunta 1. Para usted quées pensamiento sistémico.}

Se concluye que el pensamiento sistémico no se puede admitir de manera individual ni separado, es un conjunto de elementos que interactúan entre ellos como un todo, considerando las partes y el todo, comprende la totalidad de los elementos que lo constituyen; es una disciplina que busca la integración; es una forma de ser, pensar y actuar que aplica como he- rramienta para la resolución de problemas. En estas respuestas se pueden observar los dos primeros principios del pensamiento sistémico propuesto por Ackoff (2002). Los entrevistados permiten concluir que el pensamiento sistémico tiene que ver con la interacción e interrelación entre las partes de un sistema, aporta al funcionamiento de los sistemas y sus diferentes componentes, tiene relación con la forma en que un proceso afecta a otro (causa-efecto) y, permite plantear acciones de mejora.

\section{Pregunta 2. Pensamiento sistémico es.}

Con esta pregunta se busca identificar si las respuestas a la pregunta anterior están relacionadas con las definiciones de diferentes autores.

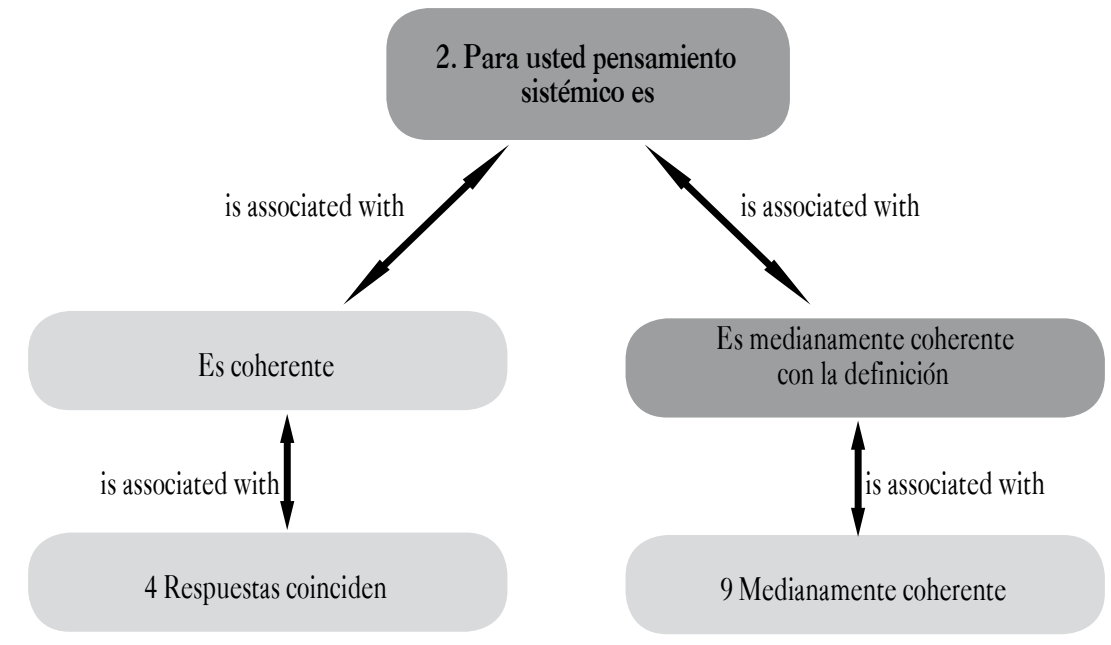

Fuente: elaboración propia (2016).

Figura 4. Pensamiento sistémico

Para facilitar el análisis se definen tres criterios: 1) es coherente, 2) es medianamente coherente con la definición y 3) no es coherente. La mayoría de los entrevistados (9 personas) dieron una respuesta medianamente coherente, para el caso, involucraron algunos elementos pero no hubo precisión en la definición de pensamiento sistémico. De otra parte, la definición de 4 entrevistados resulta coherente y no hay ninguna respuesta no coherente, lo que permite concluir que los tres grupos de entrevistados tienen conocimiento total o parcial de la temática.

Pregunta 3. Como se ha mencionado anteriormente, la pregunta 3 se focaliza segín el rol del entrevistado (docente, egresado o estudiante).

En conclusión, los docentes afirman que se incluye. Aun cuando no sea de manera directa, está en los sistemas de 
gestión. Por su parte, la mayoría de egresados aunque dicen que se incluye, precisan que no hubo demasiado desarrollo temático y, finalmente, los estudiantes tienen criterios divididos. Lo que permite ratificar la importancia de incluir de manera directa la temática en los contenidos curriculares, lo que impactaría además en las organizaciones.

Dada la experiencia de los docentes, en esta respuesta se pueden entrever los tres principios del pensamiento sistémico propuestos por Ackoff (2002). Los egresados, por su parte, hacen referencia al principio de identificar el todo contenedor del sistema. Los estudiantes no precisan ninguno de los principios de pensamiento sistémico definidos.

Pregunta 4. Desde el enfoque del convenio USTAICONTEC, ¿¿Cuál es la definición de gestión integral que considera acertada?

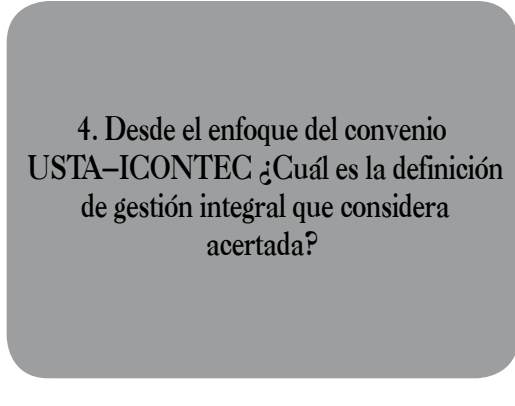

is associated with

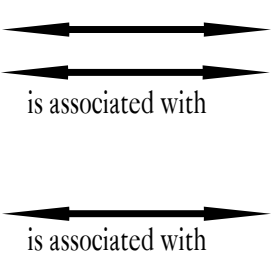

Coincide con la definición del convenio (5 citas)

Incluye definición de gestión integral y gestión integrada (7 citas)

Coincide con la definición de gestión integrada (1 cita)

Fuente: elaboración propia (2016).

Figura 5. Respuestas definición de gestión integral.

Para analizar la pregunta 4, se definen tres criterios: 1) coincide con la definición del convenio, 2) coincide con la definición de gestión integrada y, 3) incluye definición de gestión integral y de gestión integrada. Como resultado, se obtiene que la mayoría de los entrevistados eligen la opción 3, es decir, incluye definición de gestión integrada y gestión integral, seguido de los entrevistados que están de acuerdo con la definición explícita de gestión integral y, por último, únicamente un estudiante hace referencia a la definición de gestión integrada. Esto permite inferir que el concepto no se ha apropiado en su totalidad, únicamente 5 entrevistados coinciden con la definición de gestión integral desde el enfoque de la Maestría.

\section{Pregunta 5. Definición de gestión integrada.}

Los entrevistados aseveran que es un conjunto de acciones interrelacionadas para la gestión de una organización, que permite dirigir y controlar varias normas para dar cumplimiento al objetivo de esta. No tiene un eje integrador. Tiene que ver con la comprensión de los retos de las organizaciones y cómo alcanzarlos, encontrar puntos en común para planificar y encontrar acciones.

Por último, se dice que la gestión integrada tiene que ver con la correcta armonía de los elementos; la combinación de diferentes disciplinas, busca la sostenibilidad de las partes involucradas, cumplimiento de requisitos, estrategia de integralidad, calidad e intersección 
entre los sistemas. También se definió como la unión de sistemas de gestión bajo una misma autoridad para disminución de estructura y recursos.

\section{Pregunta 6. Estrategias metodológicas para involucrar el pensamiento sistémico.}

En general, a esta pregunta los docentes responden que el concepto debería involucrarse desde el principio de los programas en la estructura curricular, lograr que los estudiantes comprendan que si se tiene un enfoque sistémico, optimizan los sistemas de gestión, y establecer un diseño curricular basado en el ciclo PHVA y no por área de conocimiento. Los egresados, por su parte afirman que debe tomarse como base para el desarrollo de estrategias pedagógicas, el pensamiento sistémico como elemento integrador, tener la capacidad de abordar e integrar varios sistemas. Los estudiantes opinan que debería incluirse de manera transversal al trabajo de investigación y en diferentes asignaturas, que todos los módulos lo involucren, que no se vea de manera aislada, además como marco conceptual que permita abordar problemas.

\section{Pregunta 7. Aportes del pensamiento sistémico en las organizaciones.}

En este punto, los entrevistados realizan comentarios relacionados con el hecho de que el pensamiento sistémico contribuye en los tres ejes de sostenibilidad (económico, social y ambiental), un cambio cultural, con resultados de manera más eficiente y eficaz, permitiendo además visualizar el contexto de la organización y las partes interesadas. De igual manera, aporta en la planificación, orden, organización y verificación a través del ciclo PHVA; permite conocer procesos e interrelación y dar soluciones a problemas; permite a la organización tener una visión integrada, buscar soluciones en sistemas como calidad, ambiente, seguridad y salud en el trabajo. Por último, es una estrategia que combina visión y recursos para la obtención de resul- tados, permite ver la organización de manera holística, genera mayor claridad al momento de desarrollar un proyecto; permite articular problemas en cuanto a su identificación, elaboración estructurada y análisis para resolverlos. Igualmente, con el pensamiento sistémico se tiene una visión más acertada de los sistemas de gestión para el logro de los objetivos y sostenibilidad, aunando la solidaridad y compromiso de todas las personas de la organización.

Pregunta 8. Relación entre el pensamiento sistémico y la gestión integral.

Se enfatiza en que la totalidad de los entrevistados responden que sí existe relación entre el pensamiento sistémico y la gestión integral, hacen comentarios como que no se evidencia en la práctica, existe una brecha entre la intencionalidad, la estructura, los sistemas, etc., y la implementación. El conocimiento organizacional y la gestión del cambio están en la esencia de las normas pero no se dan en la aplicación. Incluso, hay posibilidades de crecimiento bajo la responsabilidad de los consultores y en los procesos de formación.

El pensamiento sistémico integra todas las partes y acciones necesarias para la obtención de resultados; un sistema integral es un conjunto de subsistemas, por lo tanto deberían ir de la mano como elemento integrado, además de conocer el entorno de la organización. Se agrega que el pensamiento sistémico debería ser estructurado en la formación del estudiante como ventaja competitiva, permite ver la organización de manera holística y ha sido aplicado en diferentes disciplinas, entre ellas la gestión integral. La gestión integral busca una visión total de la organización de forma sistémica, involucrando sistemas de gestión y buscando estrategias para el logro de objetivos. Se deduce la referencia de los dos primeros principios del pensamiento sistémico.

A partir del análisis de las concepciones de las partes interesadas del convenio, se concluye que sí existe 
relación entre el pensamiento sistémico y la gestión integral, conclusión coherente con la fundamentación propuesta en el objetivo 1 del presente estudio.

\section{Estrategias de enseñanza-aprendizaje empleadas actualmente para el desarrollo del pensamiento sistémico en el gestor de sistemas de gestión}

Para el desarrollo de este apartado de la investigación, se exploran los programas de especialización y maestría del convenio USTA-ICONTEC, en tal sentido se sondean los documentos maestros y fichas técnicas de los dos programas y, posteriormente, los trabajos de investigación de la línea de gestión integral, correspondientes al Programa de Maestría. Con el ánimo de contextualizar a continuación se precisan los aspectos mencionados.

Inicialmente, en el año 1993 la Universidad Santo Tomás - USTA e ICONTEC, celebran un convenio para desarrollar la Especialización en Administración y Gerencia de Sistemas de Gestión de Calidad, de este programa se graduaron más de 1800 profesionales en 86 cohortes, hasta 2015. La metodología se basa en el humanismo cristiano de la USTA y se centra en la respuesta a problemas del contexto organizacional. Posteriormente, en el año 2006, en el marco del mencionado convenio, se registra ante el Ministerio de Educación Nacional la Maestría en Calidad y Gestión Integral, que para el segundo semestre de 2014 abrió la corte 23 y en total gradúa para el mismo año a 164 profesionales (convenio USTA-ICONTEC, 2014).

\section{Proğrama de Especialización en Administración y Gerencia de Sistemas de Calidad}

Un rasgo distintivo del programa es formar implementadores que no repitan los errores que los auditores observan constantemente, y contribuir a la instauración en las organizaciones de Sistemas de Gestión de la Calidad, prácticos, útiles y que realmente contribuyan a mejorar la competitividad de las organizaciones, que además se ajuste a las características de cada organización y que sea útil para la misma, como para sus consumidores, clientes o usuarios (Convenio USTAICONTEC, 2014).

Los contenidos curriculares están formulados según tres dimensiones del aprendizaje, la dimensión gerencial y humanística permite la comprensión de las variables humanas y administrativas necesarias para gerenciar la calidad en una organización y promover una cultura ética orientada hacia el cliente y la competitividad; en esta dimensión se incluyen los módulos de planificación estratégica, liderazgo y gestión del talento humano y ética y calidad. La dimensión técnica, integra aspectos que deben conducir a la comprensión, interpretación y aplicación en diferentes contextos, de los principios, conceptos, requisitos y directrices relacionados con la gestión de la calidad; es esta dimensión están los módulos de gestión por procesos y documentación, planificación operacional, planificación y control y auditorías y mejora continua. Finalmente, la dimensión de investigación aplicada facilita el desarrollo de las habilidades para articular los conocimientos teóricos con una situación práctica; hace énfasis en los fundamentos teóricos y modelos de gestión de la calidad como ejes fundamentales del programa.

Con base en las fichas técnicas de los módulos que conforman la especialización, se realiza una matriz de análisis de contenido en la que se especificaron los siguientes elementos: 1) el tipo de módulo, 2) la dimensión 3) los objetivos y, 4) el sistema de gestión aplicable de acuerdo a las categorías definidas, que además incluyen las subcategorías propuestas (concepto, elementos y teoría abordada. A partir de esta observación se ratifica que los módulos están enmarcados en la familia de normas ISO 9001. El objetivo principal de la norma ISO 9001 es ayudar a las empresas, con el desarrollo y el funcionamiento eficaz de un sistema de 
gestión de la calidad, aumentando su capacidad para diseñar, producir y entregar productos y servicios de alta calidad; como beneficios, la norma presenta el aumento en ventas, menor número de quejas de clientes, reducción de la variación del proceso, aumento en ventajas competitivas, mayor conciencia de los empleados y mejora continua de los procesos y técnicas, que pueden servir convenientemente para implementar otros sistemas de gestión normalizados (de Olivera, 2013).

Cabe anotar que en los contenidos de la especialización no se observan de manera directa los principios relacionados con pensamiento sistémico, sin embargo, se desprenden los tres principios propuestos por Russell Ackoff (2002).

\section{Proggrama de Maestría en Calidad y Gestión In- tegral}

Es el primer programa que incursiona en el campo de la calidad y la gestión integral en el país, con el objetivo de fortalecer las competencias propias para estructurar, optimizar y articular sistemas de gestión normalizados, su denominación se especifica en el documento maestro de la Maestría, así:

La Maestría en Calidad y Gestión Integral justifica su génesis y desarrollo en cuatro grandes pilares a saber: el fenómeno de la globalización económica y su evolución hacia el concepto de desarrollo sostenible; el surgimiento de la ética empresarial a través de la denominada responsabilidad social de las organizaciones; las particularidades de las dos instituciones que conforman el convenio Universidad Santo Tomás e ICONTEC y finalmente la necesidad de la gestión de la calidad y de la gestión integral para asegurar la sostenibilidad en el tiempo de las organizaciones colombianas y latinoamericanas (Convenio USTA-ICONTEC, 2014).

En el documento maestro del mencionado programa se precisa el concepto de pensamiento sistémico propuesto por Peter Senge (2002), quien afirma que:
La esencia del nuevo papel (del administrador en una organización) será lo que llamaremos el administrador como investigador y diseñador. ¿Qué investiga? La comprensión de la organización como sistema y la comprensión de las fuerzas internas y externas que impulsan al cambio. ¿Qué diseña? Los procesos de aprendizaje por los cuales los gerentes de toda organización llegan a entender esas fuerzas y tendencias (p. 371).

Los factores diferenciadores que hacen específica la Maestría, son:

a. El manejo amplio de un concepto de calidad que incluye los aspectos tradicionalmente asociados a este concepto, es decir los relativos al cliente, a los productos que la organización entrega y a sus procesos productivos, pero que además, se extiende a la calidad de vida dentro de las organizaciones empresariales y a la de las comunidades afectadas por sus actividades productivas.

b. La investigación de nuevas prácticas administrativas que den relevancia al enfoque sistémico, a partir de la comprobada utilidad de los sistemas de gestión normalizados, para que contribuyan a que las organizaciones empresariales del país, que juegan un importante papel en la transformación de la sociedad, sean competitivas y sostenibles.

c. La búsqueda de soluciones teóricas y prácticas a la integración de los diferentes sistemas de gestión normalizados y de estos con los principios del desarrollo sostenible, para que en su conjunto contribuyan simultáneamente a la supervivencia de las organizaciones sociales y a la generación de equidad social (Convenio USTA-ICONTEC, 2014).

\section{Contenido de la Maestría}

El análisis se centra en determinar las categorías propuestas en la presente investigación: pensamiento 
sistémico, sistemas de gestión y de gestión integral incluidos en cada una de las fichas técnicas con subcategorías de concepto, elementos y teoría abordada para cada una de ellas; en general se observa que se incluyen de manera directa elementos de sistemas de gestión e indirectamente los principios pensamiento sistémico y de gestión integral. Como en el caso del Seminario de Gestión Integral que en su descripción enfatiza en que un sistema de gestión tiene que ver con la realidad de todos los aspectos de la organización, desde el aseguramiento de la calidad, incremento de la satisfacción de los clientes, protección ambiental, equilibrio con las necesidades socioeconómicas, buenas prácticas de seguridad y salud en el trabajo y, por supuesto, el aporte a la consecución de los objetivos estratégicos de la organización. De igual modo, se destaca que las organizaciones son sistemas que interactúan permanentemente y, por lo tanto, no se concibe satisfacer las expectativas de los clientes sin tener en cuenta los impactos ambientales generados por el desarrollo de sus actividades, ni considerar los riesgos aceptables para sus partes interesadas (Convenio USTA-ICONTEC, 2014).

En los contenidos de la Maestría se deduce la aplicación de los principios (Ackoff 2002) de identificar el todo contenedor, en los módulos de liderazgo y gestión del talento humano y gestión medioambiental; en el módulo de ética y responsabilidad social además se observan los dos primeros principios; en los módulos de gestión integral y globalización y efectos sociales se perciben los tres principios.

Otro de los aspectos relevantes en el análisis documental de las fichas técnicas, se evidencia en que de manera indistinta en los módulos de gestión ambiental y administración del riesgo y seminario de gestión integral, mientras que en los módulos de liderazgo, ética y responsabilidad social y gerencia estratégica se utiliza el término gestión integral y por otra parte en el módulo seminario de gestión integral en los saberes esenciales se establece el ciclo PHVA para un sistema integrado, mientras que en los saberes esenciales se hace referencia a la aplicación de un modelo de gestión integral.

Aun cuando en el documento maestro de la Maestría se precisa con claridad la definición de gestión integral, al analizar las fichas técnicas de cada uno de los módulos se observa que con mayor frecuencia se hace referencia al concepto de gestión integrada, por medio de la cual se busca integrar diferentes modelos de gestión y por ende, se hace necesario incluir esta variable en el análisis documental, con el ánimo de darle mayor claridad al concepto y en lo posible aportar conceptualmente para que no exista confusión entre las definiciones de gestión integral y gestión integrada.

\section{Relación entre el pensamiento sistémico y la ges- tión integral en el convenio USTA-ICONTEC}

Hasta el año 2015, en la Maestría de Calidad y Gestión Integral se presentan más de 150 trabajos de investigación relacionados con las diferentes líneas de profundización, de estos 38 corresponden a proyectos relacionados con la sublínea de gestión integral. Analizados los contenidos y de acuerdo con la información suministrada por el convenio, se consideran sujetos de estudio para el presente trabajo 25 proyectos de grado.

Para concluir, existen tantas definiciones como trabajos de investigación; algunos se refieren a gestión integral, otros a gestión integrada, un porcentaje representativo de los trabajos hace referencia a la teoría de Morin: "ciudadanía sistémica", algunos consideraron la teoría general de sistemas, se percibe que en algunos estudios las definiciones son equivalentes.

Encuantoa las estrategias y metodologías de investigación, en algunos trabajos se propone el sistema de gestión 
de la calidad como eje articulador, otros consideran la responsabilidad social o la gestión de riesgos, algunos aducen modelos propios (estudios Mantenimiento Productivo Total -TPM, del ICBF y del sector petróleos), la Guía PAS 55 se aplica en el proyecto de integración de sistemas de gestión de la calidad y activos fijos, finalmente, el estudio "Propuesta de un modelo de gestión integrada basado en la NTC ISO 9001:2008 y NTC 5801 para la empresa familiar Calzado Patty Sport" establece como eje articulador la estructura de alto nivel.

Se observa que en estudios como el de Velásquez y Bonilla y Martínez, se hace una descripción detallada de las estrategias de integración pero no son aplicadas a los diferentes proyectos de grado de la Maestría, los autores consideraron más conveniente aplicar modelos propios o basarse en la metodología de integración al considerar elementos comunes de las normas a integrar.

\section{Propuesta de estrategias para el desarrollo del pensamiento sistémico en los programas de forma- ción de posgrado del convenio USTA-ICONTEC}

Para proponer estrategias para el desarrollo del pensamiento sistémico en los programas del convenio, se revisan los contenidos de los tres primeros objetivos (ver figura 6). Se aplica la triangulación intrametodológica, dentro del mismo método (cualitativo), con herramientas como análisis de contenido, entrevista semiestructurada y revisión sistemática y, con base en ello, se define una ficha técnica de acuerdo a la estructura aprobada.

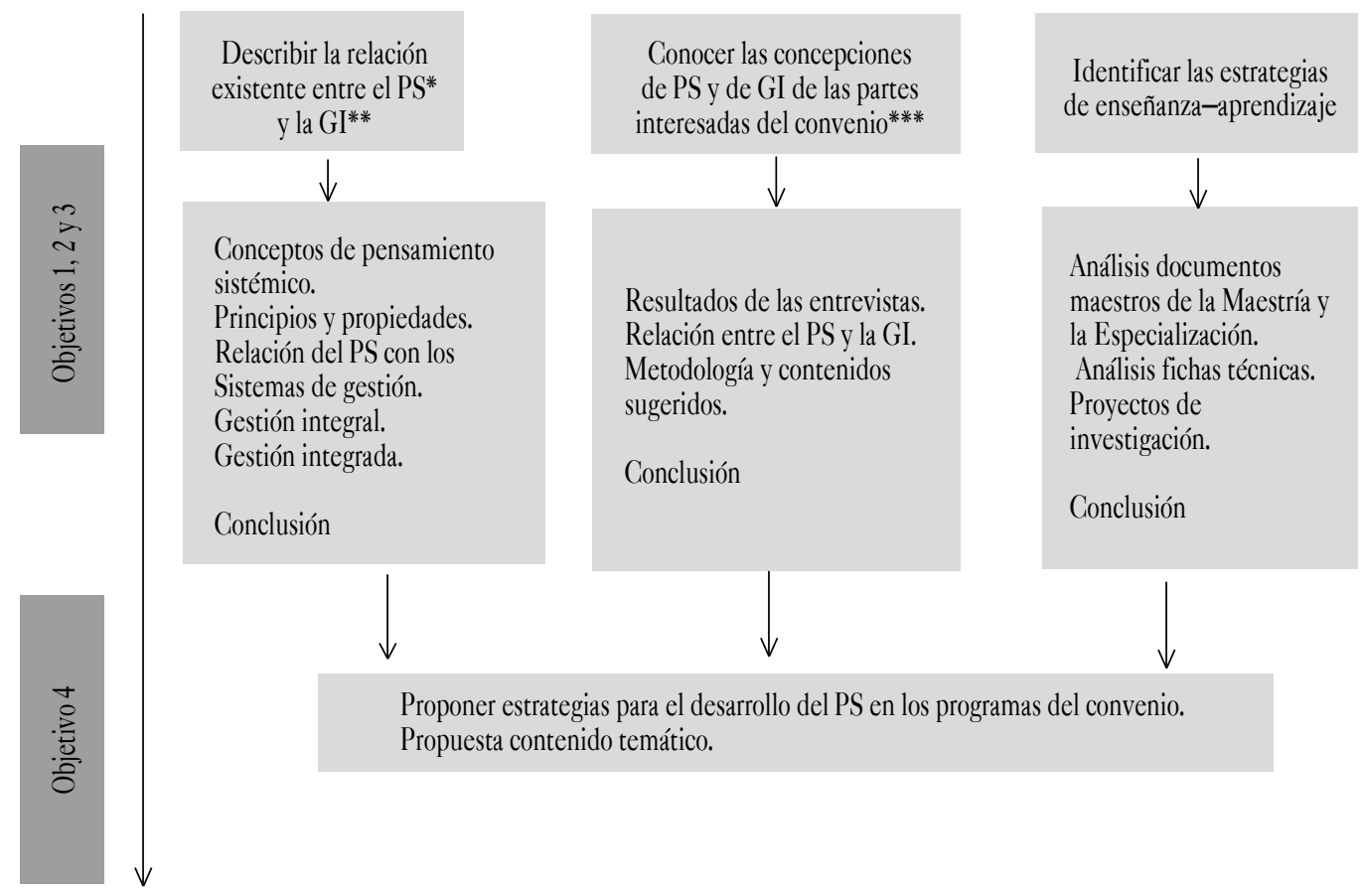

Fuente: elaboración propia (2016).

Figura 6. Elementos de entrada para propuesta de estrategias.

En la actualización curricular que está en curso, la especialización queda inmersa en la Maestría, por lo tanto, la ficha técnica es aplicable a ambos programas. 
Tabla 4. Propuesta de ficha técnica para el módulo de pensamiento sistémico.

\section{FICHA TÉCNICA \\ 1. INFORMACIÓN GENERAL}

De acuerdo a la estructura de ficha técnica aprobada por el convenio, en este punto se discrimina el programa, módulo, tipo, código, dimensión, semestre y horas presenciales, de tutoría y de trabajo individual.

\section{DESCRIPCIÓN}

A pesar de los avances tecnológicos, la globalización y el desarrollo de nuevas teorías administrativas, hoy en día muchas organizaciones conservan una estructura lineal vertical y desarrollan su objeto social según las áreas funcionales establecidas en el organigrama. El pensamiento analítico, heredado desde la Revolución Industrial, permea y para lograr la comprensión de algo en particular lo primero que se hace es dividirlo, tratando de buscar la comprensión de cada parte por separado y luego se busca integrar la comprensión de cada parte para comprender el todo, se asume que a mayor especificidad mayor comprensión. Contrario a este postulado analítico, surge el pensamiento sistémico, el cual comprende el todo y las partes además de sus interconexiones e interrelaciones. Adicionalmente, surge el concepto de administración sistémica, en el cual las organizaciones son consideradas sistemas sociales y por ende tienen estrecha relación con el medio externo y por supuesto con el interno (gestión integral). Por ello, este módulo permite precisar los conceptos de pensamiento sistémico, gestión integral y gestión integrada.

\section{OBJETIVOS}

Estudiar los conceptos del pensamiento sistémico desde la visión de diferentes autores.

Analizar los principios del pensamiento sistémico y su aplicación en la gestión de las organizaciones.

Aclarar y fortalecer el concepto de administración sistémica, en el cual la organización es considerada como un sistema social.

Clarificar el concepto de gestión integral desde el enfoque de la Maestría.

Precisar el concepto de gestión integrada y sistemas integrados de gestión.

Analizar la relación existente entre el pensamiento sistémico y la gestión integral organizacional.

\section{COMPETENCIAS DESARROLLADAS}

Tener la posibilidad de definir antecedentes, autores, teorías, principios y categorías aplicables a la gestión integral en las organizaciones como sistema social.

Conocer y aplicar las características del pensamiento sistémico en la gestión integral o en sistemas de gestión normalizados, en diferentes disciplinas.

Identificación del concepto de sistema, clarificar la diferencia entre gestión integral y gestión integrada.

Formular e implementar estrategias de integración de sistemas de gestión normalizados, basados en teorías existentes y alinearlos con los elementos del pensamiento sistémico.

Implementar sistemas de gestión integrales bajo el ciclo PHVA, alineados con la estrategia organizacional, que contribuyan al crecimiento económico y al desarrollo sostenible.

Contribuir en la aplicación de los elementos del pensamiento sistémico en el proceso de formación con el fin de que impacte en la labor de los futuros consultores, administradores y auditores de sistemas de gestión.

\section{SABERES ESENCIALES}

El docente debe asegurar que los estudiantes tengan claridad en los conceptos del pensamiento sistémico, gestión integral y gestión integrada, así como las diferencias y similitudes entre estas dos últimas variables.

Conocimiento y aplicación de los principios de pensamiento sistémico en la gestión organizacional.

Comprensión de las estrategias, metodologías y niveles de integración desde el marco normativo y otras alternativas particulares.

\section{ESTRATEGIAS METODOLÓGICAS}

Discusiones en clase, lecturas, talleres.

\section{BIBLIOGRAFÍA SUGERIDA}

Morin, E. (2015). Pensamiento complejo y educación. México D. F. En línea.

Ackoff, R. (2002). El paradigma de Ackoff. Una administración sistémica. México D. F., México: Editorial Limusa. 
Senge, P. et al. (1999). La quinta disciplina en la práctica. Barcelona, España: Editorial Granica.

O'Connor, J. y McDermott, I. (1998). Introducción al pensamiento sistémico. Barcelona, España: Urano.

Capra, F. (1998). La trama de la vida, una mueva perspectiva de los sistemas vivos. Barcelona, España: Anagrama.

Fuente: elaboración propia (2016).

\section{CONCLUSIONES}

Se reconoce el concepto de pensamiento sistémico como un sistema que es más que la suma de sus partes. Las partes propias de un sistema pueden representar otros sistemas y cada sistema por sí mismo puede ser parte de otro mayor. El pensamiento sistémico tiende a ver las cosas como partes de un todo mayor, más que como un todo que se descompone. Ackoff (2002) aborda el concepto de pensamiento sistémico en las organizaciones las cuales considera como sistemas que contienen elementos intencionados que tienen un propósito común, sistema social.

Se observa que el principio identificar un todo contenedor, del cual el objeto por explicar es una parte, es el que tiene mayor analogía entre los autores, todos lo mencionan con diferente terminología, pues hace referencia a las partes y al todo así como a las interconexiones que existen en un sistema. El principio explicar el comportamiento del todo contenedor, representa la totalidad de la información, se puede observar la similitud que guarda con el holográmico propuesto por Morin, asimismo O'Connor y McDermott afirman que un sistema cambia si se quitan o añaden piezas y Capra, al referirse que no hay partes en absoluto. El principio el comportamiento de objeto en función del todo contenedor (Ackoff, 2002), también es similar al principio de autonomía/dependencia y al principio de las partes están conectadas y funcionan todas juntas (O’Connor y McDermott, 1998).

Desde su denominación o su génesis los sistemas de gestión normalizados se diseñaron precisamente como sistemas, los principios enfoque de sistema para la ges- tión y el enfoque basado en procesos, de la norma ISO 9000:2005, tienen estrecha relación con los elementos y principios del pensamiento sistémico.

Las normas ISO poseen requisitos comunes y adoptaron el ciclo de mejora continua PHVA. Para evitar los problemas de comprensión generados, la ISO desarrolló el Anexo SL, que describió el contexto para un sistema de gestión genérico; la unificación de la estructura genera beneficios para las empresas en la implementación, para los auditores, para los consultores, además reduce costos y facilita la integración de sistemas de gestión normalizados.

La gestión integrada incorpora procesos interconectados que comparten recursos humanos, información, materiales, infraestructuras y recursos financieros con el fin de lograr un conjunto de objetivos relacionados con la satisfacción de una gran variedad de partes interesadas, se considera que la integración de los sistemas de gestión se puede definir como "la implementación de los diferentes sistemas de gestión de función específica en un único y más eficaz sistema de gestión integrado".

En desarrollo de proyectos, el modelo de pensamiento sistémico permite llevar la teoría a la práctica y generar alternativas para reducir la incertidumbre en su ejecución, el pensamiento sistémico está vinculado directamente con la cultura de la seguridad, el clima de seguridad y sistemas de gestión de seguridad y salud en el trabajo.

En el área ambiental el riesgo futuro radica en que se está ignorando el carácter sistémico en las organiza- 
ciones, en la economía y se sigue viendo al mundo de manera autónoma, se trata de manera independiente, sin tener en cuenta las interrelaciones y estructuras sistémicas del entorno y sus implicaciones en el desarrollo sostenible.

Algunos de los artículos estudiados tienen estrecha relación con el concepto de gestión integral propuesto en el convenio USTA-ICONTEC. Inicialmente Stanislav Karapetrovic, Alexandra Simón y otros autores, concluyen que además de identificar los beneficios de la integración, debe proponerse una sinergia entre los diferentes sistemas de gestión normalizados y determinarse el nivel de integración que quiere desarrollarse ya sea correspondencia, es decir con interrelaciones; comprensión, de las tareas y procesos e, integración, refiriéndose a la creación de una cultura de aprendizaje, que incluya a las partes interesadas y con una convicción de mejora continua que llegue a contribuir al desarrollo sostenible.

La teoría del pensamiento sistémico tiene una estrecha relación con la gestión integral en cuanto a que se consideran los procesos, sus interrelaciones e interconexiones así como las partes interesadas internas y externas a la organización.

El pensamiento sistémico no se puede admitir de manera individual ni separado, es un conjunto de elementos que interactúan entre ellos como un todo, considerando las partes y el todo, comprende la totalidad de los elementos que lo constituyen; es una disciplina que busca la integración; es una forma de ser, pensar y actuar que aplica como herramienta para la resolución de problemas. Lo cual es coherente con el principio identificar un todo contenedor (sistema), del cual el objeto por explicar es una parte y principio de explicar el comportamiento o propiedades del todo contenedor, propios del pensamiento sistémico.

Los docentes del convenio afirman que el pensamiento sistémico se incluye, aun cuando no sea de manera directa está en los sistemas de gestión, por su parte, la mayoría de egresados, aunque dicen que se incluye precisan que no hubo demasiado desarrollo temático y, finalmente, los estudiantes tienen criterios divididos. Lo que permite ratificar la importancia de incluir de manera directa la temática en los contenidos curriculares, lo que impactaría además en las organizaciones.

El concepto de gestión integral no se ha apropiado en su totalidad, únicamente 5 entrevistados coinciden con la definición de gestión integral desde el enfoque de la Maestría.

En cuanto a la gestión integrada, los entrevistados afirman que tiene que ver con la correcta armonía de los elementos. La combinación de diferentes disciplinas, busca la sostenibilidad de las partes involucradas, cumplimiento de requisitos, estrategia de integralidad, calidad e intersección entre los sistemas. También, se define como la unión de sistemas de gestión bajo una misma autoridad para disminución de estructura y recursos, estas apreciaciones generan confusión y no se observa total claridad en el concepto.

A partir del análisis de las concepciones de las partes interesadas, inmersas en el proceso de formación del convenio, se concluye que sí existe relación entre el pensamiento sistémico y la gestión integral, conclusión coherente con la fundamentación propuesta en el objetivo 1 del presente estudio y, por lo tanto, se presenta una propuesta para el desarrollo del pensamiento sistémico en los posgrados.

En los contenidos de la especialización no se observan de manera directa los principios relacionados con pensamiento sistémico, sin embargo, se desprenden los tres principios de pensamiento sistémico como son: a) identificar el todo contenedor (sistema) del cual el objeto por explicar es una parte, b) explicar el comportamiento o propiedades del todo contenedor y, c) explicar el comportamiento del ob- 
jeto en términos de su papel en función dentro de su todo contenedor.

En los contenidos de la Maestría se observa que se incluyen de manera directa elementos de sistemas de gestión e indirectamente los principios pensamiento sistémico y de gestión integral, como en el caso del seminario de gestión integral que en su descripción enfatiza en que un sistema de gestión tiene que ver con la realidad de todos los aspectos de la organización, desde el aseguramiento de la calidad, incremento de la satisfacción de los clientes, protección ambiental, equilibrio con las necesidades socioeconómicas, buenas prácticas de seguridad y salud en el trabajo y, por supuesto, el aporte a la consecución de los objetivos estratégicos de la organización.

En el análisis documental de las fichas técnicas se evidencia en que de manera indistinta se usan términos diferentes en los módulos de gestión ambiental y administración del riesgo y seminario de gestión integral, mientras que en los módulos de liderazgo, ética y responsabilidad social y gerencia estratégica se utiliza el término gestión integral y por otra parte, en el módulo seminario de gestión integral en los saberes esenciales se establece el ciclo PHVA para un sistema integrado, mientras que en los saberes esenciales se hace referencia a la aplicación de un modelo de gestión integral.

Existen tantas definiciones como trabajos de investigación; algunos se refieren a gestión integral, otros a gestión integrada, un porcentaje representativo de los trabajos hace referencia a la teoría de Morin "ciudadanía sistémica", algunos consideraron la teoría general de sistemas, se percibe que en algunos estudios las definiciones son equivalentes.

En cuanto a las estrategias y metodologías de investigación, en algunos trabajos se propone el sistema de gestión de la calidad como eje articulador, otros consideran la responsabilidad social o la gestión de riesgos, algunos aducen modelos propios, la Guía PAS 55 o como eje articulador la estructura de alto nivel.

Las partes interesadas relevantes de los programas de posgrado del convenio USTA-ICONTEC, es decir, las que directamente están inmersas en el proceso de formación, el cual es el que se pretende intervenir con la investigación, coincidieron en que existe relación entre el pensamiento sistémico y la gestión integral, aunque hay una brecha entre la intencionalidad, la estructura, los sistemas y la implementación; el conocimiento organizacional y la gestión del cambio están en la esencia de las normas pero no se dan en la aplicación, además, un sistema integral es un conjunto de subsistemas, por lo tanto deberían ir de la mano como elemento integrador, además de conocer el entorno de la organización.

\section{REFERENCIAS}

Abad, J., Dalmau, I., y Vilajosana, J. (2014). Taxonomic proposal for integration levels of management systems based on empirical evidence and derived corporate benefits. Journal of cleaner production, 78, 164-173.

Ackoff, R. (2002). El paradigma de Ackoff. Una administración sistémica. $1^{a}$ ed. México D. F., México: Editorial Limusa.

Ackoff, R. (2007). Conceptos básicos de la gerencia sistémica. Gotas de Conocimiento, 1-12.

Capra, F. (1998). La trama de lavida, una mueva perspectiva de los sistemas vivos. Barcelona, España: Anagrama.

Convenio USTA-ICONTEC. (2014). Documento maestro del Programa de Maestría en Calidad y Gestión Integral. Universidad Santo Tomás, Bogotá D. C., Colombia. 
De Oliveira, O. J. (2013). Guidelines for the integration of certifiable management systems in industrial companies. Journal of Cleaner Production, 57, 124-133.

Gallegos, M. (2016). Una cartografía de las ideas de la complejidad en América Latina: la difusión de Edgar Morin. [En línea]. Buenos Aires, Argentina. Recuperado de: http://www.sciencedirect.com/ science/article/pii/S1665857416300266

Hernández Sampieri, R., Fernández Collado, C., y Baptista Lucio, P. (2014). Metodología de la investigación. México D. F., México: McGraw-Hill.

ICONTEC. (2005). Sistemas de Gestión de Calidad. Fundamentos y vocabulario. NTC ISO 9000. Instituto Colombiano de Normas Técnicas y Certificación. Bogotá D. C., Colombia: ICONTEC.

ISO. (2015). Annex SL (normative) Proposals for management system standards. International Organization of Standardization. Ginebra, Suiza: ISO.

Karapetrovic, S. (2003). Musings on integrated management systems. Measuring business excellence, 7(1), 4-13.

Karapetrovic, S., y Willborn, W. (1998). Integración de la calidad y sistemas de gestión ambiental. The TQM Magasine, 10(3), 204-213. Recuperado de: https://dx.doi.org/10.1108/09544789810214800

Lara Muñoz, É. M. (2011). Fundamentos de investigación. Un enfoque por competencias. México D. F., México: Alfaomega.

Manchado, R., Tamames, S., López, M., Mohedano, L., D’Agostino, M., y Veiga de Cabo, J. (2009).
Revisiones Sistemáticas Exploratorias. Medicina y Seguridad del Trabajo, 55(216), 12-19.

Morin, E. (2015). Pensamiento complejo y educación [en línea]. México D. F., México. Recuperado de: http://www.edgarmorin.org/blog/35-educacion/387-pensamiento-complejo-y-educacion. html

O'Connor, J., y McDermott, I. (1998). Introducción al pensamiento sistémico. Barcelona, España: Ediciones Urano.

Real Academia Española. (2014). Diccionario de la lengua española 23. 'ed. Madrid, España: Espasa, 2014.

Senge P. M. (1992). La quinta disciplina. Barcelona, España: Editorial Granica.

Senge, P. et al. (1999). La quinta disciplina en la práctica. Barcelona, España: Editorial Granica.

Tejada Losada, F., y Peña Guarín, G. (2009). Reflexiones sobre las características constitutivas de la gestión integral. Revista Signos, 1(2), 87-91.

Universidad Santo Tomás. (2014a). Especialización en Administración y Gerencia de Sistemas de Calidad [en línea]. Bogotá D. C., Colombia. Recuperado de: http://especializaciongerenciasistemascalidad.usta.edu.co/index.php/informacion

Universidad Santo Tomás. (2014b). Maestría en Calidad y Gestión Integral [en línea]. Bogotá D. C., Colombia. Recuperado de: http://maestriacalidad.usta.edu.co/index. php/2011-09-05-16-43-19/informacion-general 